\title{
Type I Collagen Cleavage Is Essential for Effective Fibrotic Repair after Myocardial Infarction
}

\author{
Zengxuan Nong, ${ }^{*}$ Caroline O'Neil, ${ }^{*}$ Ming Lei, ${ }^{\dagger}$ \\ Robert Gros, ${ }^{* \dagger}$ Alanna Watson, ${ }^{* \neq}$ Amin Rizkalla, ${ }^{\S}$ \\ Kibret Mequanint, ${ }^{\S}$ Shaohua Li, ${ }^{*}$ \\ Matthew J. Frontini, ${ }^{*}$ Qingping Feng, ${ }^{\dagger}$ and \\ J. Geoffrey Pickering*¥ף|| \\ From the Robarts Research Institute* and the Departments of \\ Physiology and Pharmacology, ${ }^{\dagger}$ Biochemistry, ${ }^{\ddagger}$ Chemical and \\ Biochemical Engineering, ${ }^{,}$Medicine (Cardiology), ${ }^{\text {"ा }}$ and Medical \\ Biophysics," University of Western Ontario, London, Ontario, \\ Canada
}

Efficient deposition of type I collagen is fundamental to healing after myocardial infarction. Whether there is also a role for cleavage of type I collagen in infarct healing is unknown. To test this, we undertook coronary artery occlusion in mice with a targeted mutation $\left(\mathrm{Colla1}^{r / r}\right)$ that yields collagenase-resistant type I collagen. Eleven days after infarction, Colla1 ${ }^{r / r}$ mice had a lower mean arterial pressure and peak left ventricular systolic pressure, reduced ventricular systolic function, and worse diastolic function, compared with wild-type littermates. Infarcted $\mathrm{Colla1}^{r / r}$ mice also had greater 30-day mortality, larger left ventricular lumens, and thinner infarct walls. Interestingly, the collagen fibril content within infarcts of mutant mice was not increased. However, circular polarization microscopy revealed impaired collagen fibril organization and mechanical testing indicated a predisposition to scar microdisruption. Three-dimensional lattices of collagenase-resistant fibrils underwent cell-mediated contraction, but the fibrils did not organize into birefringent collagen bundles. In addition, time-lapse microscopy revealed that, although cells migrated smoothly on wild-type collagen fibrils, crawling and repositioning on collagenase-resistant collagen was impaired. We conclude that type I collagen cleavage is required for efficient healing of myocardial infarcts and is critical for both dynamic positioning of collagen-producing cells and hierarchical assembly of collagen fibrils. This seemingly paradoxical requirement for collagen cleavage in fibrotic repair should be considered when designing potential strategies to inhibit matrix degradation in cardiac disease. (Am J Pathol 2011, 179:2189-2198; DOI: 10.1016/j.ajpath.2011.07.017)
Rapid deposition of type I collagen is critical to cardiac repair after myocardial infarction. When effectively elaborated, a type I collagen-rich matrix provides a mechanically strong network that maintains cardiac integrity, minimizes infarct expansion, and resists maladaptive remodeling. ${ }^{1}$ Elaboration of type I collagen is a multistep process that includes expression of the genes encoding pro- $\alpha 1(\mathrm{I})$ and pro- $\alpha 2(\mathrm{I})$ collagen chains, intracellular winding of the chains, and secretion of type I procollagen. Outside the cell, the propeptide termini are cleaved and the resulting triple helical type I collagen assembles into fibrils, which then cross-link and pack. ${ }^{2}$

Collagen deposition can be opposed by processes that degrade type I collagen. Enzymes that destroy the integrity of type I collagen by cleaving the triple helical domain are important in this regard. These include the interstitial collagenases, matrix metalloproteinase (MMP)-1, MMP-8, and MMP-13, that cleave type I collagen three fourths along the length of its triple helix. Broader-spectrum MMPs, such as MMP-2 and membrane-type (MT)1-MMP/MMP-14, also have the capacity to cleave native type I collagen. ${ }^{3,4}$ Several studies have highlighted the potential for interstitial collagenases to adversely affect the heart. Transgenic expression of MMP-1 promotes ventricular dilatation and dysfunction, ${ }^{5}$ and serum levels of MMP-1 have been associated with poor patient outcomes after infarction. ${ }^{6}$ The expression of MMP- 8 has been associated with postinfarct ventricular rupture as well, ${ }^{7}$ and MMP-13 is substantially up-regulated in the failing heart. ${ }^{8}$ There is also strong evidence linking activation of broaderspectrum MMPs with adverse ventricular remodeling after infarction. ${ }^{9}$

Supported by grants from the Canadian Institutes of Health Research (FRN-11715) and the Heart and Stroke Foundation of Canada (T7081). R.G. holds a New Investigator Award from the Heart and Stroke Foundation of Canada. J.G.P. holds the Heart and Stroke Foundation of OntarioBarnett/lvey Chair

Accepted for publication July 11, 2011.

Supplemental material for this article can be found at http://ajp. amjpathol.org or at doi: 10.1016/j.ajpath.2011.07.017.

Address reprint requests to J. Geoffrey Pickering, M.D., Ph.D., London Health Sciences Centre, 339 Windermere Rd., University of Western Ontario, London, Ontario, Canada N6A 5A5. E-mail: gpickering@robarts.ca. 
Despite these findings, proof that type I collagen cleavage adversely affects healing after a myocardial infarction is lacking. Moreover, although it seems intuitive that degrading type I collagen is not desired during fibrotic myocardial repair, it is also possible that proteolytic collagen editing is important (eg, to ensure that the developing scar can productively remodel in the face of changing hemodynamic stresses). Determining whether there is a beneficial role for collagen degradation is particularly important, given that inhibiting extracellular matrix degradation is proposed as a potential therapy for myocardial infarction. ${ }^{9}$

To elucidate the role of collagenase-mediated type I collagen degradation in the healing response to myocardial infarction, we examined the consequences of coronary artery occlusion in mice expressing a collagenaseresistant form of type I collagen. These mice express a modified $\alpha 1(\mathrm{I})$ collagen chain that prevents the seminal three-fourths to one-fourth cleavage event within the type I collagen triple helix. ${ }^{10,11}$ We specifically investigated whether the scarring process was affected, using birefringence imaging, microtensile testing, and time-lapse assessment of the cell-collagen fibril interplay in vitro. We report that the ability of type I collagen to be cleaved in its triple helical domain is critical for effective fibrotic repair after myocardial infarction in mice.

\section{Materials and Methods}

\section{Transgenic Mice}

Heterozygous mice containing a targeted mutation of the col1a1 gene (B6,129-Col1a1 $\left.{ }^{\mathrm{tm} 1 \mathrm{Jae}}\right)$ were obtained from Jackson Laboratory, Bar Harbor, ME. These mice contain targeted substitutions of three amino acids at the threefourths to one-fourth collagenase cleavage site of the $\alpha 1(\mathrm{I})$ collagen chain, rendering the triple helical domain of type I collagen resistant to proteolytic cleavage. ${ }^{11} \mathrm{Ho}-$ mozygous (Col1a $1^{r / r}$ ) offspring and wild-type littermates, on the hybrid B6,129 background, were used for this study. Mice were genotyped by PCR analysis of genomic DNA harvested from the tail. ${ }^{12}$

\section{Myocardial Infarction}

Experiments were performed in accordance with protocols approved by the Animal Care and Use Committee of the University of Western Ontario, London, Ontario, Canada. Myocardial infarctions were generated in 12- to 16week-old male mice by permanent occlusion of the left anterior descending (LAD) coronary artery under general anesthesia [ketamine $(80 \mathrm{mg} / \mathrm{kg})$ and xylazine $(10 \mathrm{mg} /$ $\mathrm{kg})$ ], as described. ${ }^{13}$ As a control, sham-operated on mice were subjected to surgical anesthesia and their chests were opened, but they did not receive coronary artery ligation. Outcome analyses were undertaken by investigators (Z.N., M.L.) blinded to treatment groups and mouse genotype.

\section{Hemodynamic Assessment}

The arterial blood pressure and heart rate were recorded from anesthetized mice after cannulating the right carotid artery with a Millar-tip transducer catheter (model SPR-261, 1.4F; Millar Instruments, Inc., Houston TX). The catheter was subsequently advanced into the left ventricle (LV) for measurement of peak LV systolic pressure, LV end-diastolic pressure, maximal rate of systolic pressure development, and maximal rate of diastolic pressure decline.

\section{Tissue Harvesting and Histological Analyses}

Eleven days after surgery, mice were anesthetized and hearts were arrested in diastole by infusion of $1 \mathrm{~mL}$ of 0.1 $\mathrm{mol} / \mathrm{L} \mathrm{KCl}$ into the inferior vena cava. Hearts were then subjected to retrograde perfusion through the aorta with PBS at a pressure of $100 \mathrm{mmHg}$, followed by 3 minutes of pressurized perfusion with $4 \%$ paraformaldehyde. Excised hearts were immersion fixed in $4 \%$ paraformaldehyde for an additional 24 hours and transversely bisected at the level of the coronary ligation. The distal region was embedded in paraffin and cross-sectioned from the ligation site to the apex, obtaining 15 serial sections $(5-\mu \mathrm{m}$ thick) every $500 \mu \mathrm{m}$. Sections were stained with Picrosirius red by incubating deparaffinized sections with $0.1 \%$ Sirius red F3BA in saturated picric acid for 30 minutes and rinsing twice with $0.01 \mathrm{~mol} / \mathrm{L} \mathrm{HCl}$. Cardiac morphometry was assessed in digitally imaged sections (Northern Eclipse, Empix Imaging, Inc., Mississauga, ON, Canada). The infarct size was determined as the endocardial circumference of the infarct zone, in absolute terms and relative to that of the total left ventricular chamber, averaged from all sections obtained. The thickness of the infarct zone was determined by dividing the infarct area measurement by the midline infarct circumference from all sections. The left ventricular chamber area was based on the endocardial circumference, and the largest area served as the index of chamber size. ${ }^{14}$ The fraction of collagen in the infarct zone was quantified as the area of red staining tissue/total infarct wall area, in three low-power ( $\times 10$ objective) fields per section. Myofibroblast alignment was evaluated in the border regions of the infarct. The relative alignment of myofibroblasts was assessed from the long axis of approximately 100 nuclei within the fibrotic region zone (Northern Eclipse). The diversity of alignment was determined based on the SD of the angle distributions.

\section{Tensile Testing}

Microtensile stress tests were conducted using a computer-controlled Instron machine (model 3345; Instron Corporation, Canton, MA) at a cross-head speed of 0.5 $\mathrm{mm} /$ minute and using a $10 \mathrm{~N}$ load cell. A circumferential strip of infarct zone (width, approximately $2 \mathrm{~mm}$ ) was freshly excised from hearts 11 days after LAD coronary artery occlusion. The specimen was attached to a flat grip using Vetbond (3M Animal Care Products, St. Paul, $\mathrm{MN}$ ), and the exact cross-sectional area of each specimen was determined using a micrometer. Specimens were positioned parallel to the axis of the grip to reduce 
bending stresses. The modulus of elasticity of each specimen was calculated from the slope of the elastic portion of the stress-strain curves.

\section{Cell Culture}

Cardiac fibroblasts were isolated from the myocardium of C57BL/6 mice..$^{15}$ Briefly, minced ventricles were subjected to six 10-minute incubations in Hanks' buffer containing type II collagenase $(100 \mathrm{U} / \mathrm{mL})$ at $37^{\circ} \mathrm{C}$. Released cells were pelleted, resuspended in Dulbecco's modified Eagle's medium with $10 \%$ fetal bovine serum, and plated. After 3 hours, unattached cells were removed, adherent fibroblasts were grown for 6 to 7 days until confluence, and fibroblasts at passage two or three were studied. Human vascular smooth muscle cells (SMCs) were cultured by outgrowth from the internal thoracic artery, as previously described. ${ }^{16,17}$

\section{Collagen Isolation and Analysis of 3D Lattices}

Tails of wild-type or Col1a $1^{\text {r/r }}$ mice were dissolved in 0.1 $\mathrm{mol} / \mathrm{L}$ acetic acid and collagen purified, as previously described. ${ }^{18}$ The collagen concentration was determined spectrophotometrically (absorbance, $540 \mathrm{~nm}$ ) from Picrosirius red-stained aliquots dried onto microtiter wells. Three-dimensional (3D) collagen lattices were generated by adding a suspension of $6 \times 10^{5}$ cells $/ \mathrm{mL}$ to an equal volume of neutralized collagen solution $(2 \mathrm{mg} / \mathrm{mL})$. The mixture, $1 \mathrm{~mL}$, was then added to wells of a 24-well dish and allowed to polymerize at $37^{\circ} \mathrm{C}$ for 1 hour, before adding 1 $\mathrm{mL}$ of Dulbecco's modified Eagle's medium with 10\% fetal bovine serum. To initiate cell-mediated contraction of the lattice, gels were released from the well edges. Expression of smooth muscle $\alpha$-actin was assessed by immunostaining using a mouse monoclonal antibody (clone 1A4; DAKO Canada Inc., Burlington, ON, Canada), and the presence of collagenase-cleaved type I collagen was assessed by using a rabbit polyclonal antibody (C1-2C; IBEX Pharmaceuticals, Inc., Mount-Royal, QC, Canada).

\section{Collagen Birefringence Imaging}

Collagen organization was assessed using circular polarization microscopy. ${ }^{19}$ Picrosirius red-stained sections of myocardium and 3D collagen lattices were visualized using a Nikon Optiphot-Pol microscope (Nikon, Mississauga, ON, Canada) customized for circular polarization optics ${ }^{20}$ or an Olympus BX51 microscope (Olympus Canada, Inc., Richmond Hill, ON, Canada) with polarizerinterference filters, a liquid crystal compensator, a charge-coupled device video camera, and commercial software (Abrio LC-PolScope; Cambridge Research and Instrumentation, Inc., Hopkinton, MA). The latter system enabled measurement of light retardation by collagen, in absolute terms (nanometers), in defined fields of view. In 3D collagen lattices, 6 to 10 randomly selected cells per condition that had their long axis parallel to the section plane were studied. The mean collagen retardation was quantified in two regions of interest defined by the nuclear area, on either side of the nucleus, and corrected for the mean retardation of collagen fibrils in regions between the cells. As an additional approach that avoided cell selection, total collagen birefringence was assessed in five full fields of view (objective, $\times 60$ ) to yield the sum of retardation signals (in nanometers) for all pixels in the images. This summed value was corrected for collagen retardation in regions devoid of cells by subtracting the corresponding summed signal normalized for area. The cumulative retardation value was then divided by the total number of cells in the fields of view.

\section{Migration Analysis}

Myofibroblast and SMC motility was evaluated using digital time-lapse video microscopy. ${ }^{21-23}$ Cells plated onto dishes coated with a polymerized 3D network of mouse tail collagen $(0.8 \mathrm{mg} / \mathrm{mL})$ were imaged with an inverted microscope (Zeiss Axiovert S100; Cornwall, ON, Canada or Leica DMI6000 B; Concord, ON, Canada) using modulated contrast optics. Images were digitally acquired every 5 minutes during a 6- to 8-hour recording period, using a high-resolution charge-coupled device camera (Sony XC-75/75CE; EMPIX Imaging, Inc. or Leica DFC420 C video camera; Leica Microsystems Canada, Inc.) and time-lapse software (Northern Eclipse, Empix Imaging, Inc., and Leica Application Suite; Leica Microsystems Canada Inc.). The ambient temperature was maintained at $37^{\circ} \mathrm{C}$ using a temperature control cell [CC100 (20/20 Technology, Inc., Wilmington, NC) and LiveCell (Pathology Devices, Westminster, MD)]. To maintain physiological $\mathrm{pH}$ in room air, cells were incubated in bicarbonate-reduced medium M199 with Hanks' salts and $25 \mathrm{mmol} / \mathrm{L}$ HEPES.

\section{Statistics}

Data are expressed as mean \pm SEM. Comparisons between groups were made with the Student's $t$-test. Survival was evaluated with Kaplan-Meier analysis, and comparisons between groups were made by log-rank and Wilcoxon testing. Frequencies of mechanical microdisruption were compared using the Fisher's exact test.

\section{Results}

\section{Survival after Myocardial Infarction Is Reduced in Mice Expressing a Collagenase-Resistant}

\section{Form of Type I Collagen}

The effect of collagenase-resistant type I collagen on survival after myocardial infarction was assessed in 12- to 16-week-old Col1a $1^{\text {r/r }}$ mice and wild-type littermates. Fortysix male mice, $23 \mathrm{Col}_{1 \mathrm{a} 1^{r / /}}$ mice, and 23 wild-type littermates were subjected to permanent LAD occlusion, and survival was tracked over 1 month. One wild-type and one mutant mouse died within 24 hours of surgery. An analysis of the remaining 44 mice revealed that survival of mutant mice was significantly worse than that of control mice $(62 \%$ versus 20\% 30-day mortality; $P<0.05$; Figure 1). Two Col1a1 $1^{r / r}$ and two control mice displayed left ventricular 


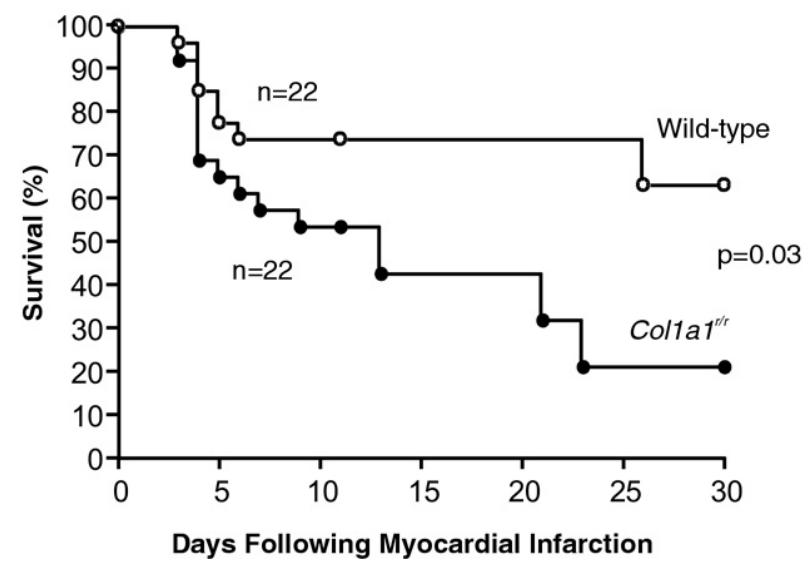

Figure 1. Survival of Colla1 $1^{r / r}$ mice and wild-type littermates after myocardial infarction. Kaplan-Meier survival curves of male Colla11 ${ }^{r / r}$ mice and male wild-type littermates over 30 days after induction of myocardial infarction by permanent LAD occlusion. $P=0.03$, Wilcoxon log-lank comparison.

rupture at death. There were no deaths in mice subjected to sham surgery. These findings suggest that the elaboration of collagenase-resistant type I collagen was not protective; instead, it reduced post-infarct survival.

\section{Cardiac Performance after Myocardial Infarction Is Reduced in Mice Producing Collagenase- Resistant Type I Collagen}

To determine whether the increased death after infarction among Col1a1 $1^{r / r}$ mice was associated with worsened cardiac performance, another 34 mice were subjected to LAD occlusion and hemodynamics were evaluated after 11 days, using a Millar-tip transducer catheter. Among sham-operated on mice, there were no differences in heart rate, mean arterial pressure, peak left ventricular systolic pressure, left ventricular contractile performance, and left ventricular diastolic performance between Col1a1 ${ }^{r / r}$ mice $(n=8)$ and wild-type littermates $(n=6)$ (Table 1). However, among mice subjected to LAD occlusion, the mean arterial pressure of Col1a $1^{r / r}$ mice $(n=$ 9 ) was $34.9 \%$ lower than that of wild-type mice $(n=11)$ $(P<0.01)$. Left ventricular systolic pressure in Col1a $1^{r / r}$ mice was $23.5 \%$ lower $(P<0.05)$, left ventricular con- tractile performance was $34.4 \%$ lower $(P<0.05)$, and left ventricular diastolic performance was $31.2 \%$ lower $(P<$ 0.05). Thus, the inability to cleave type I collagen at the three-fourths to one-fourth site adversely affected both systolic and diastolic left ventricular performance after myocardial infarction.

\section{Ventricular Distortion after Myocardial Infarction Is Worsened in Mice Producing Collagenase- Resistant Type I Collagen}

Cardiac morphometry was assessed in Picrosirius redstained sections of hearts arrested in diastole and pressure fixed 11 days after infarction or sham surgery. No differences in left ventricular chamber size or wall thickness were found between Col1a1 ${ }^{r / r}$ and control mice subjected to a sham operation (Figure 2). After LAD occlusion, the infarct territory in wild-type mice occupied $36 \% \pm 1.7 \%$ of the midventricular myocardium, and this was not different in mutant mice $(34 \% \pm 2.7 \%, P=0.49)$. However, the infarct zone was significantly thinner, attenuated by $26.8 \%(P<$ 0.01 , Figure 2) and the absolute length of the infarct zone was greater in mutant than in wild-type mice $(6.8 \pm 0.3$ versus $5.2 \pm 0.4 \mathrm{~mm} ; P<0.01)$. Also, the left ventricular chamber size was greater $(48.7 \%, P<0.01)$.

These morphometric data raised the possibility that the healing infarct in Col1a1/r mouse hearts was predisposed to expansion. To further assess this, fresh segments of scar zone 11 days after infarction were excised from the infarct territory and subjected to tensile testing, using a $10 \mathrm{~N}$ load cell. The modulus of elasticity was $2.6 \pm 0.7 \mathrm{MPa}$ in scar segments from wild-type hearts $(n=4)$ and $1.5 \pm 0.4 \mathrm{MPa}$ in scars from Col1a1 $1^{r / r}$ hearts $(n=5, P=0.22)$, suggesting greater elasticity of mutant scars. The presence of abrupt, but transient, losses of stretch-induced tension in the scars from mutant hearts, well before total mechanical failure was reached (Figure 3), was more striking, suggesting microdisruptions in the tissue as it was stretching. This phenomenon was observed in all five mutant infarcts studied (1.2 microfailures per scar) but only in one of four wild-type infarcts $(0.25$ microfailures per scar, $P<0.05)$. These findings indicate that the infarct zone of mice expressing collagenase-resistant type I collagen has a reduced ability to resist tensile forces.

Table 1. Hemodynamic Parameters in WT and Col1a1 $1^{r / r}$ Mice after Myocardial Infarction

\begin{tabular}{|c|c|c|c|c|}
\hline \multirow[b]{2}{*}{ Parameter } & \multicolumn{2}{|c|}{ Sham-operated on mice } & \multicolumn{2}{|c|}{ LAD-occluded mice } \\
\hline & $\mathrm{WT}(n=6)$ & Col1a1 ${ }^{r / r}(n=8)$ & $\mathrm{WT}(n=11)$ & Col1atr/r $(n=9)$ \\
\hline Heart rate (beats/minute) & $393 \pm 8$ & $392 \pm 9$ & $420 \pm 13$ & $391 \pm 9$ \\
\hline $\mathrm{MAP}(\mathrm{mmHg})$ & $87 \pm 3$ & $87 \pm 6$ & $86 \pm 5$ & $56 \pm 4^{*}$ \\
\hline LVSP (mmHg) & $109 \pm 4$ & $112 \pm 5$ & $106 \pm 8$ & $81 \pm 4^{*}$ \\
\hline LVEDP (mmHg) & $4.2 \pm 0.6$ & $3.7 \pm 0.4$ & $11.6 \pm 2.1^{\dagger}$ & $7.9 \pm 0.7^{\dagger}$ \\
\hline $\mathrm{LV} \mathrm{dP} / \mathrm{dt}_{\max }(\mathrm{mmHg} / \mathrm{second})$ & $7973 \pm 484$ & $7689 \pm 353$ & $6916 \pm 453$ & $4607 \pm 441^{*}$ \\
\hline $\mathrm{LV}-\mathrm{dP} \mathrm{dt}_{\max }(\mathrm{mmHg} /$ second $)$ & $8171 \pm 533$ & $8404 \pm 400$ & $6159 \pm 290^{\dagger}$ & $4239 \pm 380^{*}$ \\
\hline
\end{tabular}

Values are given as mean \pm SEM.

${ }^{\star} P<0.05$ vs sham and WT LAD occluded.

${ }^{\dagger} P<0.05$ vs sham.

$\mathrm{dP} / \mathrm{dt}_{\max }$, maximum rate of increase in left ventricular systolic pressure; $-\mathrm{dP}_{\mathrm{dt}} \mathrm{d}_{\text {max }}$, maximum rate of decline in left ventricular diastolic pressure; LVEDP, left ventricular end-diastolic pressure; LVSP, peak left ventricular systolic pressure; MAP, mean artery pressure; WT, wild type. 
A

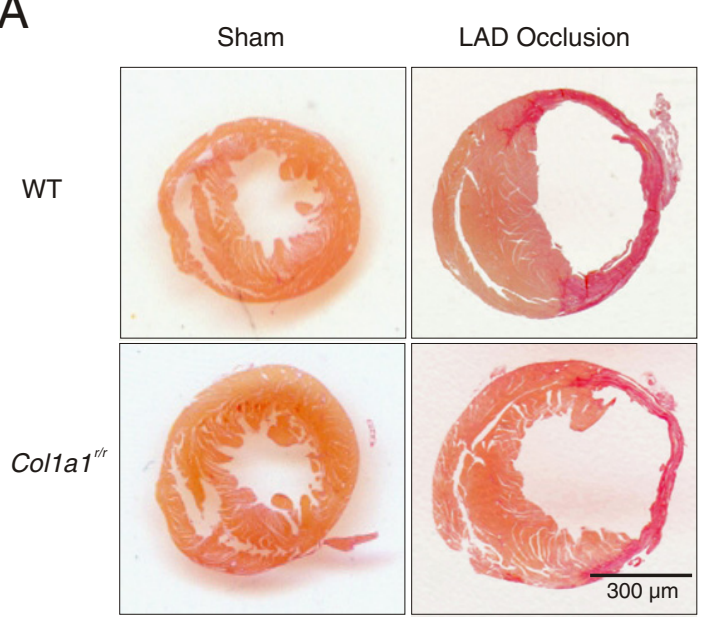

$\mathrm{B}$
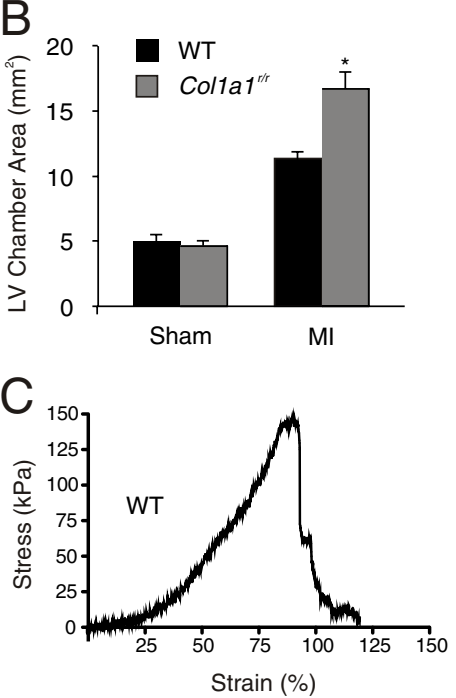
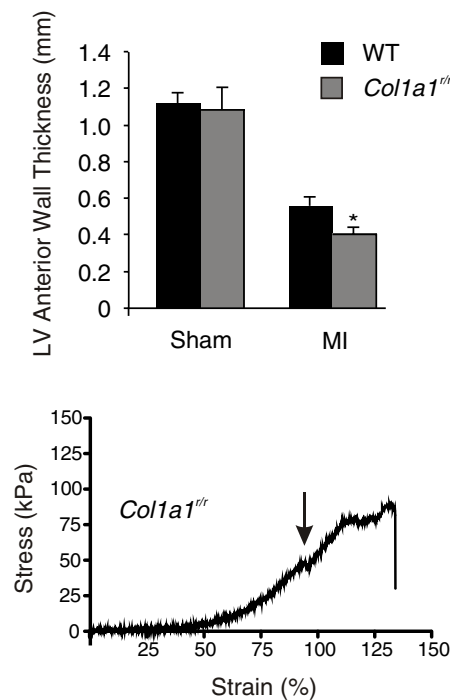

Figure 2. Impaired left ventricular remodeling after myocardial infarction (MI) in Col1a $1^{r / r}$ mice. A: Picrosirius red-stained cross sections of pressure-fixed hearts arrested in diastole 11 days after sham surgery or LAD occlusion. B: There was a greater LV chamber area and a thinner infarct territory in Col1a ${ }^{r / r}$ mice after MI compared with wild-type (WT) mice. No differences existed in sham-operated on hearts. ${ }^{*} P<0.05$. C: Stress-strain plots of the scar zone harvested 11 days after LAD occlusion in a WT (left) or a Col1a1 $1^{r / r}$ mutant (right) mouse. The plot for the mutant scar reveals greater elasticity and a small abrupt decline in the generated stress in the otherwise linear portion of the curve (arrow), indicating a microdisruption event.

\section{Healing Infarct Zone of Col1a $1^{r / r}$ Mice Displays Abnormal Collagen Fibril Organization}

We next evaluated the fibrotic response in the hearts. Collagen content in hearts subjected to sham surgery was not different between wild-type and mutant mice $(4.6 \% \pm 0.04 \%$ versus $4.9 \% \pm 0.5 \%)$. Interestingly, the collagen content within the infarct zone was also not different between wild-type and mutant mice $(50.8 \% \pm$ $5.8 \%$ versus $54.2 \% \pm 4.4 \%$ ), indicating that resistance to collagen cleavage did not lead to an accumulation in collagen fibers. In contrast, distinctions in collagen fibril morphological features were noted. This was examined using circularly polarized light, an approach that clearly
A
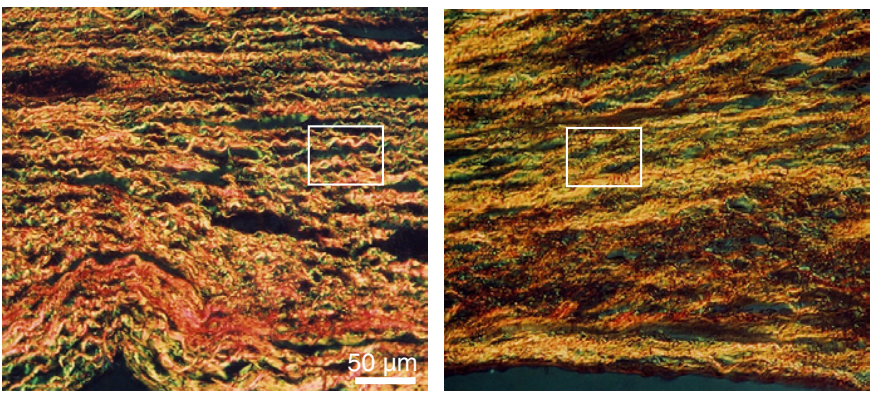

B

WT

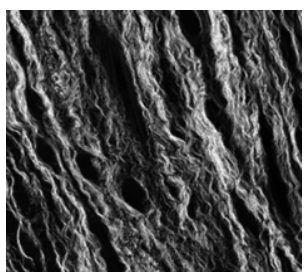

Col1a1 $1^{\text {rt }}$

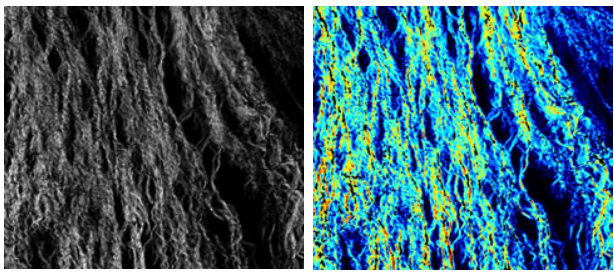

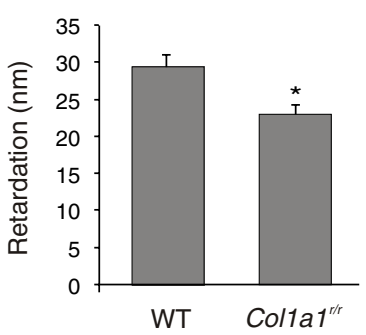

Figure 3. Collagen fibers in the infarct zone of Col1a $^{r / r}$ mice display less order. A: Circular polarization microscopy images of Picrosirius red-stained sections of the LV, 11 days after LAD occlusion. Co-aligned bundles of birefringent collagen fibers are seen in the infarct zone of wild-type (WT) mice (left), whereas in Col1a $1^{\text {r/t }}$ mice, fibers were more diffusely oriented and thinner (middle). Insets (right): Well-delineated wavy appearance of collagen fibers (arrow) in the infarct zone of WT mice only (top panel, arrow) versus those in mutant mice (bottom panel). B: Digitally acquired retardation images of the infarct border zone of mice after LAD occlusion, imaged with polarized light and liquid crystal compensation. The resulting retardation images (left) are independent of illumination intensity, such that images can be directly compared. The corresponding heat maps (right) depict reduced retardation in the infarct zone of mutant mice, despite copious collagen fibers having been deposited. Quantitative data from 11 mutant and 9 WT littermate mice are shown. ${ }^{*} P<0.05$. 
delineates fibril morphological features by innate collagen birefringence enhanced by the Picrosirius red stain. Collagen fibrils in the infarct zone of mice expressing wild-type type I collagen appeared as well-aligned, circumferentially oriented bands of thick fibers with a wavy sinusoidal pattern (Figure 3A). In contrast, in the infarct zone of Col1a $11^{r / r}$ mice, collagen fibers were thinner and less organized. Moreover, these fibers typically did not display sinusoidal periodicity (Figure 3A). These differences were particularly evident in the middle circumferential one third of the scar and the infarct borders.

Collagen birefringence increases as its molecular, supramolecular, and fibrillar orders increase, structural changes that increase optical anisotropy. ${ }^{19,20,24}$ Therefore, we quantified collagen retardation as an indicator of collagen organization within the fibrotic tissue. Retardation of collagen fibers in noninfarcted hearts was not different between wild-type and mutant mice ( $4.9 \pm 0.7$ versus $4.4 \pm 0.7 \mathrm{~nm} ; P=0.55$ ). However, in mice subjected to infarction, the mean collagen retardation within fibrotic regions was $18.0 \%$ lower in hearts of Col1a $1^{r / r}$ compared with wild-type mice (Figure 3B). Together, these morphological and birefringence findings indicate that the inability to efficiently cleave type I collagen did not affect collagen accumulation after infarction but significantly impaired the ability of elaborated collagen to hierarchically organize.

\section{Collagenase-Resistant Collagen Fails to Assemble into High-Ordered Fibrils in Response to Cell-Mediated Contractile Forces}

To determine whether the reduced collagen organization was directly related to collagenase resistance, we evaluated the interplay between mesenchymal cells and collagen fibrils in a 3D network. ${ }^{25}$ Collagen fibril lattices were prepared from collagen harvested from tails of wildtype or Col1a1 $1^{r / r}$ mice and embedded with mouse myocardial fibroblasts. Networks embedded with human vascular SMCs were similarly prepared, in view of the myofibroblast-like attributes of these cells. When collagen fibril lattices are released from the sides of the culture dish, they contract, in association with elaboration of collagen-degrading enzymes. ${ }^{26}$ We observed that both mouse and human mesenchymal cells contracted the collagen gels and that contraction did not occur if cells were not present in the network. Interestingly, networks generated from collagenase-resistant collagen contracted more than those constructed of wild-type collagen $(63 \% \pm 4 \%$ versus $46 \% \pm 3 \%$ at 48 hours; $P<0.05$; Figure 3). Immunostaining using an antibody specific for collagenase-cleaved type I collagen revealed a signal in fibrils immediately adjacent to a few cells $(<10 \%)$, suggesting highly localized collagen editing with a transient cleavage product (Figure 4). Signal was not detected in gels of mutant collagen. The proportion of fibroblasts in mutant collagen that expressed immunodetectable smooth muscle $\alpha$-actin was not different from that for fibroblasts in wild-type collagen $(33.2 \% \pm 2.5 \%$ versus $33.8 \% \pm 2.2 \%)$, suggesting no differences in the intrinsic
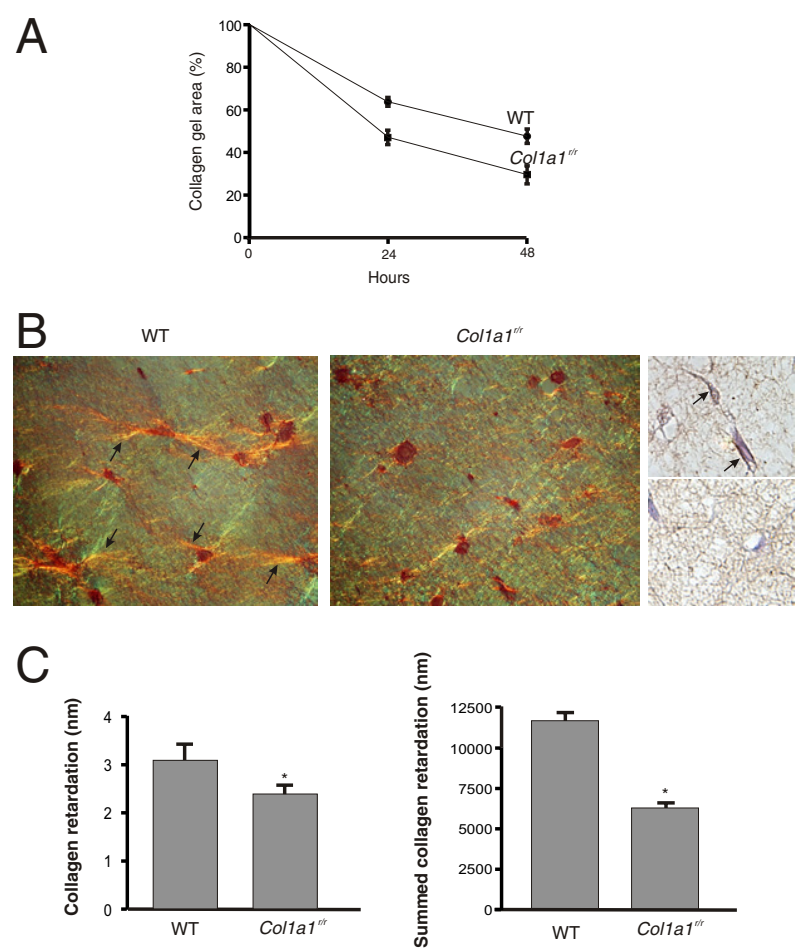

Figure 4. Cell-mediated contraction of collagen fibril lattice and assembly of pericellular collagen fibrils in vitro. A: The magnitude of contraction of collagen lattices embedded with mouse cardiac fibroblasts and constructed of either collagenase-resistant collagen or wild-type (WT) fibrils. B: Sections from cell-embedded collagen fibril lattices stained after 48 hours with Picrosirius red and viewed with circular polarized light. Birefringent collagen fibrils assembled on the surface of cell body and projections in the WT collagen network (arrows). In contrast, only a modest accumulation of birefringent fibrils can be seen around the cells in the network of collagenase-resistant collagen. Immunostaining at 24 hours using the $\mathrm{C} 1-2 \mathrm{C}$ antibody revealed evidence for collagenase-cleaved type I collagen in immediate pericellular regions in WT (arrows, top right panel), but not mutant (bottom right panel), gels. C: Collagen fibril retardation of circularly polarized light. Retardation was quantified as the average retardation in defined regions of interest associated with selected cells (left) and the average cell-associated collagen retardation from the entire unselected field of view, and expressed as summed retardation (right). ${ }^{*} P<0.05$.

contractile properties of the cells. Collectively, these data suggest a degree of collagen fibril disruption and/or slippage during the contraction process that did not proceed in gels of mutant collagen.

To evaluate the architecture of the fibrils, lattices were examined histologically and fibril retardation was quantified by polarization microscopy. Before contraction, both wild-type and mutant fibrils appeared as relatively homogeneous lattices of fibrils (thickness, $<0.2 \mu \mathrm{m}$ ). However, after 48 hours of contraction, the collagen fibrils in the immediate vicinity of the cells had substantially remodeled into thicker (1 to $8 \mu \mathrm{m}$ ) and highly birefringent bundles of fibrils (Figure 4). This fibril reorganization was observed for wild-type collagen but markedly less so for collagenase-resistant collagen (Figure 4B). The mean retardation of pericellular collagen fibrils was $29.0 \%$ higher in gels of wild-type collagen, when evaluated on fibrils surrounding randomly selected individual cells, and $47.7 \%$ higher when assessed as the cumulative retardation index, normalized for background and the number of cells in the section (Figure 4C). Taken together, these 
findings establish that, in response to cell-mediated contractile forces, collagen fibrils organize into high-ordered bundles and that this assembly process requires the proteolytic cleavage of the type I collagen triple helix.

\section{Cell Migration and Positional Alignment Are Inhibited by Collagenase-Resistant Collagen Fibrils}

The ability of collagen-producing cells to crawl into the wound and appropriately orient also determines the efficacy of fibrotic healing. To determine whether collagenase-resistant collagen affected fibroblast/myofibroblast positioning after infarction, we quantified the alignment of mesenchymal cells in the infarct border zones, the site of fibroblast/myofibroblast streaming. This revealed a $61.5 \%(P<0.01)$ greater dispersion in fibroblast orientation in the hearts of Col1 $11^{r / r}$ mice compared with that in infarcted hearts of wild-type mice (Figure 5A). This dispersed alignment suggests a reduced ability of the infiltrating mesenchymal cells to co-align in an environment of collagenase-resistant collagen.

To further evaluate motility in a collagen fibril environment, we undertook time-lapse video microscopy of cells on crawling along 3D lattices of wild-type or mutant type I collagen. Mouse myocardial fibroblasts migrated along a network of wild-type collagen, with a migration speed at $32.9 \pm 4.5 \mu \mathrm{m} /$ hour, and human SMCs migrated at $19.4 \pm 3.3 \mu \mathrm{m} /$ hour. However, the migration speeds of either mouse or human cells were significantly lower when they crawled on collagenase-resistant collagen fibrils $(P<0.01$, Figure $5 \mathrm{~B})$.

Tracking the migration paths of individual cells revealed striking differences in the patterns of migration. As illustrated in Figure 5, C and D, fibroblasts on wild-type collagen typically translocated in a given direction for one or more cell lengths and then turned and migrated in another direction. This pattern reflects the lack of an external chemotactic gradient but also indicates an ability of the cells to quickly reorient as they explore their environment. In contrast, cells on collagenase-resistant collagen displayed only minor shifts in the position of the cell centroid and little change in the overall direction of crawling. Moreover, although cells on wild-type collagen glided smoothly over the fibrils, on mutant collagen they exhibited a halting translocation pattern. This was associated with stretching and, at times, bending of the trailing aspect of the cell, which appeared to effectively with-
A

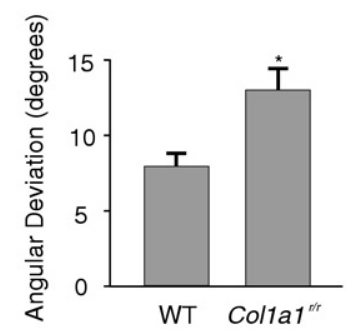

D
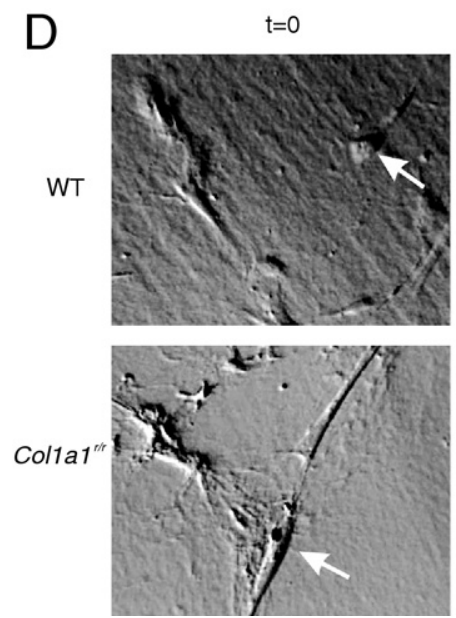

B

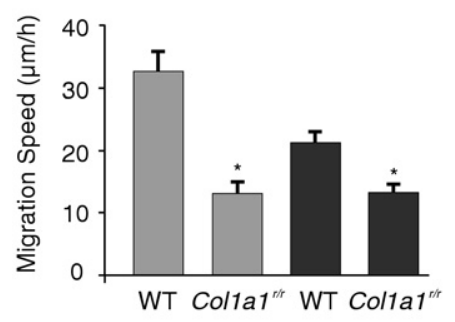

C
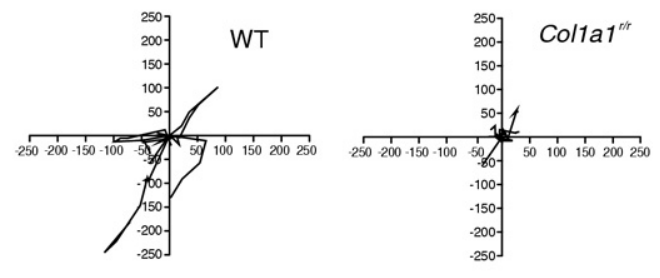

$t=100 \min$
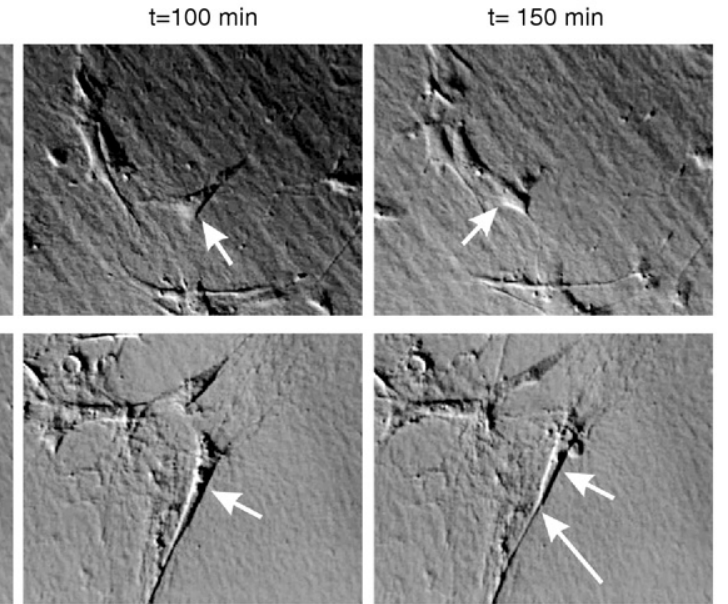

Figure 5. Cell motility and dynamic repositioning are suppressed on collagenase-resistant collagen. A: The SD of alignment of fibroblasts/myofibroblasts within the transition zone of infarcted myocardium 11 days after LAD occlusion in control and Col1a $1^{r / r}$ mice. B: The migration speed of mouse myocardial fibroblasts (light gray) and human SMCs (dark gray) crawling on a 3D collagen fibril lattice derived from the tails of either wild-type (WT) or Col1a ${ }^{r / r}$ mice. C: Spider plots of mouse fibroblasts crawling on the designated collagen lattices. Tracings depict the hourly translocation of the cell centroid over 6 hours. D: Images of individual cells crawling on collagen fibrils over 150 minutes. Arrows (top panel): A cell that translocates in a given direction and then turns to reorient approximately 90 degrees from its original path. Arrows (bottom panel): A cell in which the leading edge protrudes forward but the rear aspect does not release from the substrate, resulting in stretching of the cell body and tail. The long arrow denotes the development of a bend in the stretched tail, suggesting additional restriction to cell turning or reorientation. These distinctions in the dynamic motility patterns of cells on WT and mutant collagen can be seen in Supplemental Videos S1 and S2 (available at http://aip.ampathol.org). ${ }^{*} P<0.05$ versus the corresponding WT value. 
hold the cell from changing position and orientation (Figure 5D; see also Supplemental Video S1 at http:// aip.ampathol.org). The mutant collagen network itself was seen to fold and compress underneath the cells, indicating that the cells were generating contractile forces necessary for migration but were incapable of releasing from the collagen substrate to effectively translocate (see Supplemental Video S2 at http://aip.ampathol.org). These findings reveal a collagen cleavage-based cellular release phenomenon that is necessary for collagen-producing cells to efficiently translocate and reorient in their environment.

\section{Discussion}

We have studied the role of type I collagen cleavage in the healing process after myocardial infarction in mice. The findings reveal that mice that produce a collagenaseresistant form of type I collagen undergo maladaptive ventricular remodeling after myocardial infarction, with suppressed cardiac performance and increased mortality compared with littermate control mice. These adverse events were associated with impaired hierarchical ordering of the collagen fibrils deposited in the infarct zone. We also established that mesenchymal cells that were otherwise fully capable of assembling collagen fibrils into coherent bundles could not execute this function effectively with collagenase-resistant collagen fibrils. Moreover, the ability of mesenchymal cells to crawl, orient, and co-align during tissue remodeling was impaired in an environment of collagenase-resistant collagen. These findings suggest that, despite the potential to weaken a developing scar, proteolysis of type I collagen is required for key steps in fibrotic healing after myocardial infarction.

Col1a $1^{r / r}$ mice contain a targeted mutation in the Col1a1 gene that eliminates the Gly775-Ile776 cleavage site located approximately three fourths along the length of the triple helical domain of type I collagen. This is an important proteolysis site because, once cleaved, the collagen triple helix unfolds, rendering the collagen chains susceptible to further proteolytic degradation. Several enzymes are capable of mediating this cleavage, and several, including MMP-8, MMP-13, and MMP-14, are expressed after myocardial infarction. $8,27,28$ Thus, Col1a $1^{r / r}$ mice constitute a powerful tool for elucidating the role of collagen degradation during infarct healing, and their use circumvents compensation or redundancy among collagen-degrading enzymes. In addition, collagenases can have targets other than type I collagen. ${ }^{9}$ Therefore, the findings observed with Col1a $1^{r / r}$ mice reflect only the altered type I collagen and not enzymatic cleavage states of other protein targets. Furthermore, because type I collagen in Col1a $1^{r / r}$ mice can still be cleaved at a secondary $\mathrm{N}$-telopeptide site, ${ }^{11}$ the current findings specifically establish the importance of three-fourths to one-fourth triple helix cleavage in infarct repair.

Previous studies have established that Col1a $1^{\text {r/r }}$ mice have a propensity to accumulate excess collagen fibers. Aged Col1a $1^{r / r}$ mice accumulate collagen fibers in the skin, ${ }^{11}$ and increased fibrosis has been found in atherosclerotic lesions ${ }^{29}$ and aneurysmal arteries. ${ }^{30}$ Given these findings, it is noteworthy that collagen accumulation in the LV of Col1a1/r mice after infarction was not greater than that in control mice. This finding is consistent with a previous report ${ }^{31}$ and probably reflects the fact that the balance of production and degradation of type I collagen after acute myocardial injury is heavily weighted to the production side. However, the current findings also establish that, despite this production bias, collagen degradation plays a key role in the infarct healing process. This role was evidently not to control the amount of collagen deposited but to regulate the architecture of the deposited fibrils. Qualitatively, wavy collagen fibers typical of well-organized fibrous tissue emerged in the infarct zone of control mice but generally not in the fibrotic territory of Col1a1/r/ mice. Quantitatively, fibrils in the hearts of Col1a $1^{r / r}$ mice were less birefringent and had a reduced ability to retard circularly polarized light. Thus, the hierarchical collagen ordering process fundamental to fibrotic healing requires three-fourths to one-fourth collagen cleavage.

The poor response to myocardial infarction in Col1a $1^{1 / r}$ mice could reflect a primary defect in collagen organization in the infarct zone or a secondary effect of a generalized perturbation in cardiovascular function in Col1a ${ }^{r / r}$ mice. Although the latter possibility cannot be excluded, we did not identify baseline cardiovascular abnormalities in the Col1a1/r mice studied. Heart rate, mean arterial pressure, peak left ventricular systolic pressure, left ventricular chamber size, and left ventricular wall thickness were similar in sham-operated on wild-type and mutant mice. Thus, for example, baseline differences in afterload are an unlikely explanation for the worsened response of Col1a $1^{r / r}$ mice to infarction. Pre-infarction collagen fibril content and organization were also not different between mutant and wild-type mice.

Therefore, we propose that the maladaptive post-infarct remodeling in Col1a $1^{r / r}$ mice was because of the elaboration of mutant collagen in the infarct zone, resulting in impaired fibrotic healing. This was supported by mechanical testing, which identified a predisposition of the scar zone in mutant mice to tension-induced microfailure. Moreover, in vitro analyses identified a direct relationship between the ability of collagen to undergo cleavage and collagen fibril assembly. There may also be modifier genes in the B6,129 strain used that heightened the impact of the collagen mutation in vivo. Mice with a 129 background have been found to have more vulnerable infarct healing than inbred C57BL/6 mice $^{32}$ and greater activation of the renin-angiotensin system. ${ }^{33}$

Our 3D culture analyses identified two components of tissue remodeling that depend on collagenase-competent collagen fibrils. The first component is the phenomenon of cell contraction-mediated assembly of collagen fibrils. We identified that collagen fibril assembly proceeds at the cell surface and found that this fiber-building process required collagen fibrils that can be cleaved at the three-fourths to one-fourth proteolysis site. The fact that collagen fibril birefringence decreased when collagen cleavage was abrogated seems paradoxical but 
suggests a process by which site-specific collagen cleavage enables collagen fibrils to reassemble into thicker fibrils. The fact that contraction of mutant collagen gels was greater than that of wild-type gels further suggests that this augmented fibril assembly process is based on a highly functional collagen slippage phenomenon. Therefore, we propose that type I collagen fibrils productively organize through a cleave-slide-and-reassemble cascade and that this cascade is critical to optimizing fibrosis after myocardial infarction.

The second process for which collagen fibril cleavage was determined to be critical was optimizing the motility and positioning of the collagen-producing cell. We found that a 3D network of type I collagen fibrils was a suitable substrate for motility and enabled a smooth, gliding migration pattern with efficient shifting of cell orientation. However, on collagenase-resistant collagen, cell migration was slow and halting, with marked stretching of the trailing aspect of the cell. Moreover, there was accentuated deformation and compression of the surrounding collagen network. These findings suggest a role for collagen cleavage in loosening cell-substrate connections to allow the cell body and tail to efficiently and appropriately follow the leading edge. Previous studies ${ }^{22,34}$ have suggested that products of type I collagen cleavage may be promigratory. Collagen cleavage might also be important for ensuring that other promigratory matrix molecules, such as minor collagens or fibronectin, can appropriately interact with the cell. Collagen synthesis is suppressed in cells on or in a 3D lattice ${ }^{18,35}$; thus, in this system, the dominant target for editing is likely pre-existing collagen fibrils. In vivo, cleavage of recently deposited collagen may also be relevant, particularly because collagen deposition and maturation during wound healing can proceed over days to weeks. ${ }^{20,36}$

The ability of fibroblasts to quickly reorient and finetune their direction of traveling within remodeling tissue could serve two purposes. First, it could ensure that the cell can explore and test its new environment efficiently. Second, there may be a need for cells to rapidly shift position in response to ongoing structural changes and mechanical forces, a circumstance highly relevant to the remodeling infarct zone. ${ }^{36}$ We found that in hearts expressing collagenase-resistant collagen there was reduced alignment of myofibroblasts entering the infarct zone. Because fibroblast/myofibroblast alignment defines collagen fibril alignment, this impaired ability to reorient could be an additional means by which the fidelity of scar formation was reduced.

In summary, despite the potential deleterious consequences of degrading collagen fibrils, cleavage of type I collagen is required for effective fibrotic healing after myocardial infarction in mice. Both the hierarchical assembly of collagen fibrils and the positioning of the collagen-elaborating cells depend on the capacity with which type I collagen can be cleaved. The seemingly paradoxical requirement of type I collagen cleavage for cardiac repair should be considered when designing therapeutic strategies to block matrix degradation in the heart.

\section{References}

1. Jugdutt BI: Ventricular remodeling after infarction and the extracellular collagen matrix: when is enough enough? Circulation 2003, 108 : 1395-1403

2. Prockop DJ, Kivirikko KI, Tuderman L, Guzman NA: The biosynthesis of collagen and its disorders. N Engl J Med 1979, 301:13-23, 77-85

3. Aimes RT, Quigley JP: Matrix metalloproteinase-2 is an interstitia collagenase: inhibitor-free enzyme catalyzes the cleavage of collagen fibrils and soluble native type I collagen generating the specific 3/4- and 1/4-length fragments. J Biol Chem 1995, 270:5872-5876

4. Ohuchi E, Imai K, Fujii Y, Sato H, Seiki M, Okada Y: Membrane type 1 matrix metalloproteinase digests interstitial collagens and other extracellular matrix macromolecules. J Biol Chem 1997, 272:24462451

5. Kim HE, Dalal SS, Young E, Legato MJ, Weisfeldt ML, D'Armiento J: Disruption of the myocardial extracellular matrix leads to cardiac dysfunction. J Clin Invest 2000, 106:857-866

6. Soejima H, Ogawa H, Sakamoto T, Miyamoto S, Kajiwara I, Kojima S, Hokamaki J, Sugiyama S, Yoshimura M, Suefuji H, Miyao Y, Fujimoto $\mathrm{K}$, Miyagi $\mathrm{H}$, Kishikawa $\mathrm{H}$ : Increased serum matrix metalloproteinase-1 concentration predicts advanced left ventricular remodeling in patients with acute myocardial infarction. Circ J 2003, 67:301-304

7. van den Borne SW, Cleutjens JP, Hanemaaijer R, Creemers EE, Smits JF, Daemen MJ, Blankesteijn WM: Increased matrix metalloproteinase- 8 and -9 activity in patients with infarct rupture after myocardial infarction. Cardiovasc Pathol 2009, 18:37-43

8. Spinale FG, Coker ML, Heung LJ, Bond BR, Gunasinghe HR, Etoh T, Goldberg AT, Zellner JL, Crumbley AJ: A matrix metalloproteinase induction/activation system exists in the human left ventricular myocardium and is upregulated in heart failure. Circulation 2000, 102: 1944-1949

9. Spinale FG: Myocardial matrix remodeling and the matrix metalloproteinases: influence on cardiac form and function. Physiol Rev 2007, 87:1285-1342

10. Wu H, Byrne MH, Stacey A, Goldring MB, Birkhead JR, Jaenisch R, Krane SM: Generation of collagenase-resistant collagen by site-directed mutagenesis of murine pro alpha $1(\mathrm{I})$ collagen gene. Proc Nat Acad Sci U S A 1990, 87:5888-5892

11. Liu X, Wu H, Byrne M, Jeffrey J, Krane S, Jaenisch R: A targeted mutation at the known collagenase cleavage site in mouse type I collagen impairs tissue remodeling. J Cell Biol 1995, 130:227-237

12. Zhao W, Byrne MH, Boyce BF, Krane SM: Bone resorption induced by parathyroid hormone is strikingly diminished in collagenase-resistant mutant mice. J Clin Invest 1999, 103:517-524

13. Feng Q, Lu X, Jones DL, Shen J, Arnold JM: Increased inducible nitric oxide synthase expression contributes to myocardial dysfunction and higher mortality after myocardial infarction in mice. Circulation 2001, 104:700-704

14. Gao XM, Dart AM, Dewar E, Jennings G, Du XJ: Serial echocardiographic assessment of left ventricular dimensions and function after myocardial infarction in mice. Cardiovasc Res 2000, 45:330-338

15. Subramanian SV, Kelm RJ, Polikandriotis JA, Orosz CG, Strauch AR: Reprogramming of vascular smooth muscle alpha-actin gene expression as an early indicator of dysfunctional remodeling following heart transplant. Cardiovasc Res 2002, 54:539-548

16. Pickering JG, Weir L, Rosenfield K, Stetz J, Jekanowski J, Isner JM: Smooth muscle cell outgrowth from human atherosclerotic plaque: implications for the assessment of lesion biology. J Am Coll Cardiol 1992, 20:1430-1439

17. Isner JM, Kearney M, Bauters C, Leclerc G, Pickering JG, Reissen R, Weir L: Use of human tissue specimens obtained by directional atherectomy to study restenosis. Trends Cardiovasc Med 1994, 4:213-221

18. Rocnik E, Saward L, Pickering JG: HSP47 expression by smooth muscle cells is increased during arterial development and lesion formation and is inhibited by fibrillar collagen. Arterioscler Thromb Vasc Biol 2001, 21:40-46

19. Whittaker P, Kloner RA, Boughner DR, Pickering JG: Quantitative assessment of myocardial collagen with picrosirius red staining and circularly polarized light. Basic Res Cardiol 1994, 89:397-410 
20. Pickering JG, Ford CM, Chow LH: Evidence for rapid accumulation and persistently disordered architecture of fibrillar collagen in human coronary restenosis lesions. Am J Cardiol 1996, 78:633-637

21. Rocnik EF, Chan BMC, Pickering JG: Evidence for a role of collagen synthesis in arterial smooth muscle cell migration. J Clin Invest 1998, 101:1889-1898

22. Li S, Chow LH, Pickering JG: Cell surface-bound collagenase-1 and focal substrate degradation stimulate the rear release of motile vascular smooth muscle cells. J Biol Chem 2000, 275:35384-35392

23. Fera E, O'Neil C, Lee W, Li S, Pickering JG: Fibroblast growth factor-2 and remodeled type I collagen control membrane protrusion in human vascular smooth muscle cells: biphasic activation of Rac1. J Biol Chem 2004, 279:35573-35582

24. Pickering JG, Boughner DR: Fibrosis in the transplanted heart and its relation to donor ischemic time: assessment with polarized light microscopy and digital image analysis. Circulation 1990, 81:949-958

25. Grinnell F: Fibroblasts, myofibroblasts, and wound contraction. J Cell Biol 1994, 124:401-404

26. Unemori EN, Werb Z: Reorganization of polymerized actin: a possible trigger for induction of procollagenase in fibroblasts cultured in and on collagen gels. J Cell Biol 1986, 103:1021-1031

27. Wilson EM, Moainie SL, Baskin JM, Lowry AS, Deschamps AM, Mukherjee R, Guy TS, St John-Sutton MG, Gorman JH 3rd, Edmunds LH Jr, Gorman RC, Spinale FG: Region- and type-specific induction of matrix metalloproteinases in post-myocardial infarction remodeling. Circulation 2003, 107:2857-2863

28. Peterson JT, Li H, Dillon L, Bryant JW: Evolution of matrix metalloprotease and tissue inhibitor expression during heart failure progression in the infarcted rat. Cardiovasc Res 2000, 46:307-315

29. Fukumoto Y, Deguchi JO, Libby P, Rabkin-Aikawa E, Sakata Y, Chin MT, Hill CC, Lawler PR, Varo N, Schoen FJ, Krane SM, Aikawa M:
Genetically determined resistance to collagenase action augments interstitial collagen accumulation in atherosclerotic plaques. Circulation 2004, 110:1953-1959

30. Deguchi JO, Huang H, Libby P, Aikawa E, Whittaker P, Sylvan J, Lee RT, Aikawa M: Genetically engineered resistance for MMP collagenases promotes abdominal aortic aneurysm formation in mice infused with angiotensin II. Lab Invest 2009, 89:315-326

31. Lindsey ML, Yoshioka J, MacGillivray C, Muangman S, Gannon J, Verghese A, Aikawa M, Libby P, Krane SM, Lee RT: Effect of a cleavage-resistant collagen mutation on left ventricular remodeling. Circ Res 2003, 93:238-245

32. Gao XM, Xu Q, Kiriazis H, Dart AM, Du XJ: Mouse model of postinfarct ventricular rupture: time course, strain- and gender-dependency, tensile strength, and histopathology. Cardiovasc Res 2005, 65:469-477

33. Wang Q, Hummler E, Nussberger J, Clément S, Gabbiani G, Brunner HR, Burnier M: Blood pressure, cardiac, and renal responses to salt and deoxycorticosterone acetate in mice: role of Renin genes. J Am Soc Nephrol 2002, 13:1509-1516

34. Pilcher BK, Dumin JA, Sudbeck BD, Krane SM, Welgus HG, Parks WC: The activity of collagenase- 1 is required for keratinocyte migration on a type I collagen matrix. J Cell Biol 1997, 137:14451457

35. Eckes B, Mauch C, Huppe G, Krieg T: Downregulation of collagen synthesis in fibroblasts within three-dimensional collagen lattices involves transcriptional and posttranscriptional mechanisms. FEBS Lett 1993, 318:129-133

36. van den Borne SW, Diez J, Blankesteijn WM, Verjans J, Hofstra L, Narula J: Myocardial remodeling after infarction: the role of myofibroblasts. Nat Rev Cardiol 2010, 7:30-37 